\title{
2D Dependency Pairs for Proving Operational Termination of CTRSs
}

\author{
Salvador Lucas ${ }^{1,2(\bowtie)}$ and José Meseguer ${ }^{2}$ \\ 1 DSIC, Universitat Politècnica de València, València, Spain \\ ${ }^{2}$ Computer Science Department, University of Illinois at Urbana-Champaign, \\ Champaign, IL, USA \\ slucas@dsic.upv.es
}

\begin{abstract}
The notion of operational termination captures nonterminating computations due to subsidiary processes that are necessary to issue a single 'main' step but which often remain 'hidden' when the main computation sequence is observed. This highlights two dimensions of nontermination: one for the infinite sequencing of computation steps, and the other that concerns the proof of some single steps. For conditional term rewriting systems (CTRSs), we introduce a new dependency pair framework which exploits the bidimensional nature of conditional rewriting (rewriting steps + satisfaction of the conditions as reachability problems) to obtain a powerful and more expressive framework for proving operational termination of CTRSs.
\end{abstract}

Keywords: Conditional term rewriting $\cdot$ Dependency pairs $\cdot$ Program analysis $\cdot$ Operational termination

\section{Introduction}

Assume that we have an interpreter for a logic $\mathcal{L}$, i.e., an inference machine that, given a theory $\mathcal{S}$ and a goal formula $\varphi$, will try to incrementally build a proof tree for $\varphi$. Intuitively, we call $\mathcal{S}$ terminating if for any $\varphi$ the interpreter either finds a proof in finite time, or fails in all possible attempts also in finite time. The notion of operational termination captures this idea, meaning that, given an initial goal, an interpreter will either succeed in finite time in producing a closed proof tree, or will fail in finite time, not being able to close or extend further any of the possible proof trees, after exhaustively searching all such proof trees [12]. In particular, operational termination captures a 'vertical' dimension of the termination behavior which is missing in the usual definition of termination of relations as well-founded, i.e., "without infinite reduction sequences" (the 'horizontal' dimension).

Research partially supported by NSF grant CNS 13-19109. Salvador Lucas' research was developed during a sabbatical year at the CS Dept. of the UIUC and was also partially supported by Spanish MECD grant PRX12/00214, MINECO project TIN201021062-C02-02, and GV grant BEST/2014/026 and project PROMETEO/2011/052.

(C) Springer International Publishing Switzerland 2014

S. Escobar (Ed.): WRLA 2014, LNCS 8663, pp. 195-212, 2014.

DOI: $10.1007 / 978-3-319-12904-4 \_11$ 
Available tools for proving operational termination of conditional rewriting (AProVE [10] or VMTL [16]) rely on transformations $\mathcal{U}$ that map each operational termination problem for the CTRS $\mathcal{R}$ into a termination problem for a TRS $\mathcal{U}(\mathcal{R})$. Then, available methods for proving termination of $\mathcal{U}(\mathcal{R})$ are used. However, this transformational approach has substantial limitations.

Example 1. Consider the following CTRS $\mathcal{R}$ [15, Example 8]

$$
\begin{aligned}
h(d) & \rightarrow c(a) \\
h(d) & \rightarrow c(b) \\
f(k(a), k(b), x) & \rightarrow f(x, x, x) \\
g(x) & \rightarrow k(y) \Leftarrow h(x) \rightarrow d, h(x) \rightarrow c(y)
\end{aligned}
$$

As reported in [15, Example 8], $\mathcal{U}(\mathcal{R})$ is not terminating. However, our methods in this paper will show that $\mathcal{R}$ is operationally terminating (Example 19).

Most termination tools for proving termination of (variants of) rewriting with TRSs implement extensions or generalizations of the Dependency Pair Framework $[7,8]$. The main idea is the following: the rules $\ell \rightarrow r$ that are able to produce infinite sequences are those whose right-hand side $r$ contains (possibly recursive) function calls. The calls associated to $\ell \rightarrow r$ are represented as new rules $u \rightarrow v$, that are collected in a new $\operatorname{TRS} \operatorname{DP}(\mathcal{R})$ of dependency pairs (DPs); $\mathcal{R}$ and $\operatorname{DP}(\mathcal{R})$ determine dependency chains whose finiteness characterize termination of $\mathcal{R}[1]$.

In this paper we generalize this approach to deterministic 3-CTRSs, which are the basis of rewriting-based languages like CafeOBJ [5] or Maude [3]. In Sect. 3 we show that computations starting from minimal operationally nonterminating terms can always follow a precise path where two sources of nontermination can be identified: infinite sequences of rewriting steps (an horizontal dimension), and infinitely many attempts to check the satisfaction of the conditions in the rules (a vertical dimension). Section 4 introduces a definition of dependency pairs that makes such a bidimensional nature of infinite computations explicit (we call them 2D DPs). The corresponding notion of chain of dependency pairs permits a completely independent treatment of both dimensions of the termination problems. For 2-CTRSs (a subclass of 3-CTRSs), we characterize termination (i.e., the absence of infinite rewrite sequences) in terms of the "horizontal" component of our 2D DPs only. In Sect. 5, we adapt the Dependency Pair Framework [7,8] to mechanize proofs of operational termination of deterministic 3-CTRSs using 2D DPs. The framework can also be used to prove termination of 2-CTRSs which are not operationally terminating.

Example 2. The following deterministic 2-CTRS $\mathcal{R}$ :

$$
\begin{aligned}
g(a) & \rightarrow c(b) \\
b & \rightarrow f(a) \\
f(x) & \rightarrow x \Leftarrow g(x) \rightarrow c(y)
\end{aligned}
$$




\begin{tabular}{|c|c|}
\hline (Refl) & $\overline{t \rightarrow \rightarrow^{*} t}$ \\
\hline & $u \rightarrow^{*} t$ \\
\hline (Tran) & $s \rightarrow^{*} t$ \\
\hline (Cong) & $\begin{array}{c}s_{i} \rightarrow t_{i} \\
f\left(s_{1}, \ldots, s_{i}, \ldots, s_{k}\right) \rightarrow f\left(s_{1}, \ldots, t_{i}, \ldots, s_{k}\right) \\
\text { for all } f \in \mathcal{F} \text { and } 1 \leq i \leq k=\operatorname{ar}(f)\end{array}$ \\
\hline (Repl) & $\begin{array}{l}\qquad \begin{array}{c}\sigma\left(s_{1}\right) \rightarrow^{*} \sigma\left(t_{1}\right) \ldots \sigma\left(s_{n}\right) \rightarrow^{*} \sigma\left(t_{n}\right) \\
\sigma(\ell) \rightarrow \sigma(r)\end{array} \\
\text { for all rules } \ell \rightarrow r \Leftarrow s_{1} \rightarrow t_{1} \cdots s_{n} \rightarrow t_{n} \in \mathcal{R} \\
\text { and substitutions } \sigma .\end{array}$ \\
\hline
\end{tabular}

Fig. 1. Inference rules for conditional rewriting

is not operationally terminating. However, it is terminating. We can prove both things in our framework (see Examples 13 and 15), illustrating its expressiveness.

Section 6 develops the framework by introducing a number of processors and illustrating their use. Section 7 discusses related work and concludes.

\section{Preliminaries}

Recall from [14] the usual notions and notations regarding term rewriting and CTRSs. An (oriented) CTRS $\mathcal{R}$ is a pair $\mathcal{R}=(\mathcal{F}, R)$ where $\mathcal{F}$ is a signature and $R$ a set of rules $\ell \rightarrow r \Leftarrow s_{1} \rightarrow t_{1}, \cdots, s_{n} \rightarrow t_{n}$, where the conditions $s_{i} \rightarrow t_{i}$ for $1 \leq i \leq n$ are intended to express the reachability of (instances of) $t_{i}$ from (instances of) $s_{i}$. As usual, $\ell$ and $r$ are called the left- and right-hand sides of the rule, and the sequence $s_{1} \rightarrow t_{1}, \cdots, s_{n} \rightarrow t_{n}$ (often abbreviated to $c$ ) is the conditional part of the rule. Rewrite rules $\ell \rightarrow r \Leftarrow c$ are classified according to the distribution of variables among $l, r$, and $c$, as follows: type 1, if $\mathcal{V}$ ar $(r) \cup \mathcal{V}$ ar $(c) \subseteq \mathcal{V}$ ar $(\ell)$; type 2, if $\mathcal{V} a r(r) \subseteq \mathcal{V} a r(\ell)$; type 3, if $\mathcal{V} a r(r) \subseteq \mathcal{V} a r(\ell) \cup \mathcal{V} a r(c)$; and type 4, if no restriction is given. An $n$-CTRS contains only rewrite rules of type $m \leq n$. An oriented 3-CTRS $\mathcal{R}$ is called deterministic if for each rule $\ell \rightarrow r \Leftarrow s_{1} \rightarrow$ $t_{1}, \ldots, s_{n} \rightarrow t_{n}$ in $\mathcal{R}$ and each $1 \leq i \leq n$, we have $\mathcal{V} a r\left(s_{i}\right) \subseteq \mathcal{V} \operatorname{Var}(l) \cup \bigcup_{j=1}^{i-1} \mathcal{V} \operatorname{ar}\left(t_{j}\right)$. Given $\mathcal{R}=(\mathcal{F}, R)$, we consider $\mathcal{F}$ as the disjoint union $\mathcal{F}=\mathcal{C} \uplus \mathcal{D}$ of symbols $c \in \mathcal{C}$ (called constructors) and symbols $f \in \mathcal{D}$ (called defined functions), where $\mathcal{D}=\{\operatorname{root}(l) \mid(l \rightarrow r \Leftarrow c) \in R\}$ and $\mathcal{C}=\mathcal{F}-\mathcal{D}$. Terms $t \in \mathcal{T}(\mathcal{F}, \mathcal{X})$ such that $\operatorname{root}(t) \in \mathcal{D}$ are called defined terms. $\operatorname{Pos}_{\mathcal{D}}(t)$ is the set of positions $p$ of subterms $\left.t\right|_{p}$ such that $\operatorname{root}\left(\left.t\right|_{p}\right) \in \mathcal{D}$.

A conditional rewrite $s \rightarrow^{*} t$ with a CTRS $\mathcal{R}$ exists if and only if it has a closed proof tree using the inference system in Fig. 1. We say that a proof tree $T$ is closed whenever it is finite and contains no open goals; it is well-formed if 
it is either an open goal, or a closed proof tree, or a derivation tree of the form $\frac{T_{1} \quad \ldots \quad T_{n}}{G}$ where, for each $j, T_{j}$ is itself well-formed, and there is $i \leq n$ such that $T_{i}$ is not closed, for any $j<i T_{j}$ is closed, and each of the $T_{i+1}, \ldots, T_{n}$ is an open goal [12]. An infinite proof tree is well-formed if it is an ascending chain of well-formed finite proof trees. Intuitively, well-formed trees are the trees that an interpreter would incrementally build when trying to solve one condition at a time from left to right. We write $s \rightarrow_{\mathcal{R}} t$ (resp. $\left.s \rightarrow_{\mathcal{R}}^{*} t\right)$ iff there is a wellformed proof tree for $s \rightarrow t$ (resp. $s \rightarrow^{*} t$ ). The CTRS $\mathcal{R}$ is called operationally terminating if no infinite well-formed tree for a goal $s \rightarrow_{\mathcal{R}} t$ or $s \rightarrow_{\mathcal{R}}^{*} t$ exists. The CTRS $\mathcal{R}$ is called terminating if there is no infinite sequence $t_{1} \rightarrow_{\mathcal{R}} t_{2} \rightarrow_{\mathcal{R}} \cdots$.

\section{Minimal Operationally Nonterminating Terms in CTRSs}

Given a proof tree $T, \operatorname{root}(T)$ is the formula (goal) at the root of the tree, and left $(G)$ is the left-hand side $s$ of goal $G$, where $G$ is $s \rightarrow t$ or $s \rightarrow^{*} t$ for some terms $s$ and $t$.

Definition 1 (Operationally Nonterminating Term). Let $\mathcal{R}$ be a CTRS. A term $t$ such that left $(\operatorname{root}(T))=t$ for an infinite well-formed proof tree $T$ is called operationally nonterminating. If there is no infinite well-formed proof tree $T$ such that left $(\operatorname{root}(T))=t$, then we call $t$ operationally terminating.

Definition 2 (Minimality). Let $\mathcal{R}$ be a CTRS. An operationally nonterminating term $t$ is called minimal if every strict subterm $u$ of $t$ (i.e., $t \triangleright u$ ) is operationally terminating. Let $\mathcal{T}_{o p-\infty}$ be the set of minimal operationally nonterminating terms associated to $\mathcal{R}$.

The following lemma shows that operationally nonterminating terms always contain a minimal operationally nonterminating term.

Lemma 1. Let $\mathcal{R}=(\mathcal{F}, R)$ be a CTRS and $s \in \mathcal{T}(\mathcal{F}, \mathcal{X})$. If $s$ is operationally nonterminating, then there is a subterm $t$ of $s(s \unrhd t)$ such that $t \in \mathcal{T}_{o p-\infty}$.

Proposition 1 below establishes that, for $t \in \mathcal{T}_{\text {op- } \infty}$, there is a precise way for an infinite computation to proceed. Roughly speaking, a rule $\ell \rightarrow r \Leftarrow \bigwedge_{i=1}^{n} s_{i} \rightarrow t_{i}$ must be used to try a root-step on a reduct of $t$. Then, there is a minimal operationally nonterminating subterm which is either (1) an instance of a nonvariable subterm of the right-hand side $r$ of the rule (so that the infinite computation continues through the horizontal dimension), or (2) an instance of a non-variable subterm of one of the left-hand sides $s_{i}$ of a condition $s_{i} \rightarrow t_{i}$ (the infinite computation continues through the vertical dimension). Given a term $t$, $\mathcal{D} \operatorname{Subterm}(\mathcal{R}, t)=\left\{\left.t\right|_{p} \mid p \in \mathcal{P} s_{\mathcal{D}}(t)\right\}$ is the set of defined subterms of $t$ with respect to rules in $\mathcal{R}$. Let $\operatorname{DRules}(\mathcal{R}, t)$ be the set of (possibly conditional) rules in $\mathcal{R}$ defining root $(t)$ which depend on other defined symbols in $\mathcal{R}$ :

$$
\operatorname{DRules}(\mathcal{R}, t)=\{\ell \rightarrow r \Leftarrow c \in \mathcal{R} \mid \operatorname{root}(\ell)=\operatorname{root}(t), r \notin \mathcal{T}(\mathcal{C}, \mathcal{X})\} .
$$

The dependency is captured as $r \notin \mathcal{T}(\mathcal{C}, \mathcal{X})$ in the above definition. 
Example 3. For $\mathcal{R}$ in Example 1, DRules $(\mathcal{R}, h(x))=\emptyset$ (because $c(a), c(b) \in$ $\mathcal{T}(\mathcal{C}, \mathcal{X})), \operatorname{DRules}(\mathcal{R}, g(x))=\emptyset($ again $k(y) \in \mathcal{T}(\mathcal{C}, \mathcal{X}))$ and $\operatorname{DRules}(\mathcal{R}, f(x, x, x))$ $=\{(3)\}$.

For each $v \in \mathcal{D} \operatorname{Subterm}(\mathcal{R}, r), \operatorname{DRules}(\mathcal{R}, v)$ contains the rules that will (eventually) be used in root steps $\sigma(\ell) \rightarrow \sigma(r)$ for some $\ell \rightarrow r \Leftarrow c \in \operatorname{DRules}(\mathcal{R}, v)$ in the immediate continuation of the infinite computation in the horizontal dimension (starting from an instance $\sigma(v)$ of $v$ ). With regard to the vertical dimension, given a term $t$, the set of 'proper' conditional rule defining $\operatorname{root}(t)$ is:

$$
\operatorname{Rules}_{C}(\mathcal{R}, t)=\left\{\ell \rightarrow r \Leftarrow \bigwedge_{i=1}^{n} s_{i} \rightarrow t_{i} \in \mathcal{R} \mid \operatorname{root}(\ell)=\operatorname{root}(t), n>0\right\} .
$$

These are the rules involved in transitions of computations to upper levels. We let $\operatorname{URules}(\mathcal{R}, t)=\operatorname{DRules}(\mathcal{R}, t) \cup$ Rules $_{C}(\mathcal{R}, t)$ to be the set of used rules.

Example 4. For $\mathcal{R}$ in Example 1, URules $(\mathcal{R}, h(x))=\operatorname{DRules}(\mathcal{R}, h(x))$ and $\operatorname{URules}(\mathcal{R}, f(x, x, x))=\operatorname{DRules}(\mathcal{R}, f(x, x, x))$. However $\operatorname{URules}(\mathcal{R}, g(x))=$ $\operatorname{DRules}(\mathcal{R}, g(x)) \cup$ Rules $_{C}(\mathcal{R}, g(x))=\{(4)\}$.

Proposition 1. Let $\mathcal{R}$ be a deterministic 3-CTRS. Then, for all $t \in \mathcal{T}_{\text {op- } \infty}$, there exist $\alpha: \ell \rightarrow r \Leftarrow \bigwedge_{i=1}^{n} s_{i} \rightarrow t_{i}$ and a substitution $\sigma$ such that $t \stackrel{>\Lambda}{\longrightarrow} \sigma(\ell)$, and there is a term $v$ such that $\ell \not v, \sigma(v) \in \mathcal{T}_{o p-\infty}$ and either

1. $\alpha \in \operatorname{DRules}(\mathcal{R}, t)$, for all $1 \leq i \leq n, \sigma\left(s_{i}\right)$ is operationally terminating and $\sigma\left(s_{i}\right) \rightarrow^{*} \sigma\left(t_{i}\right)$, and $v \in \mathcal{D} \operatorname{Subterm}(\mathcal{R}, r)$ is such that $\operatorname{URules}(\mathcal{R}, v) \neq \emptyset$, or

2. $\alpha \in$ Rules $_{C}(\mathcal{R}, t)$, there is $i, 1 \leq i \leq n$ such that $\sigma\left(s_{j}\right)$ is operationally terminating and $\sigma\left(s_{j}\right) \rightarrow^{*} \sigma\left(t_{j}\right)$ for all $j, 1 \leq j<i$, and $v \in \mathcal{D} \operatorname{Subterm}\left(\mathcal{R}, s_{i}\right)$ is such that $\operatorname{URules}(\mathcal{R}, v) \neq \emptyset$.

Remark 1. In the following we do not impose that the domain of the substitutions be finite. This is usual practice in the dependency pair approach, where a single substitution is used to instantiate an infinite number of variables coming from renamed versions of the dependency pairs (see below).

The next result formalizes a bidimensional view of infinite computations starting from minimal operational nonterminating terms: they can be viewed as a path over $\mathbb{N} \times \mathbb{N}$, where each bidimensional point $\left(x_{i}, y_{i}\right)$ is labeled with a rule $\alpha_{i}$.

Theorem 1. Let $\mathcal{R}=(\mathcal{F}, R)$ be a deterministic $3-C T R S$ and $t \in \mathcal{T}_{\text {op- } \infty}$. There is a substitution $\sigma$ and an infinite sequence $\left\{\left(x_{i}, y_{i}, \alpha_{i}\right)\right\}_{i \in \mathbb{N}}$ of triples $\left(x_{i}, y_{i}, \alpha_{i}\right) \in$ $\mathbb{N} \times \mathbb{N} \times R$ such that, for all $i \geq 0, x_{i+1}+y_{i+1}=x_{i}+y_{i}+1$ and

1. $x_{0}=y_{0}=0, \alpha_{0} \in \operatorname{URules}(\mathcal{R}, t)$ and $t \stackrel{>\Lambda}{\longrightarrow} * \sigma\left(\ell_{0}\right)$.

2. For all $i \geq 0$, and $\alpha_{i}: \ell_{i} \rightarrow r_{i} \Leftarrow \bigwedge_{j=1}^{n_{i}} s_{j}^{i} \rightarrow t_{j}^{i} \in R$, we have $\sigma\left(\ell_{i}\right) \in$ $\mathcal{T}_{o p-\infty}$; furthermore, there is a term $v_{i}$ such that $\ell_{i} \not v_{i}, \sigma\left(v_{i}\right) \in \mathcal{T}_{o p-\infty}$, $\sigma\left(v_{i}\right) \stackrel{>\Lambda}{\longrightarrow} \sigma\left(\ell_{i+1}\right), \alpha_{i+1} \in \operatorname{URules}\left(v_{i}\right)$, and 


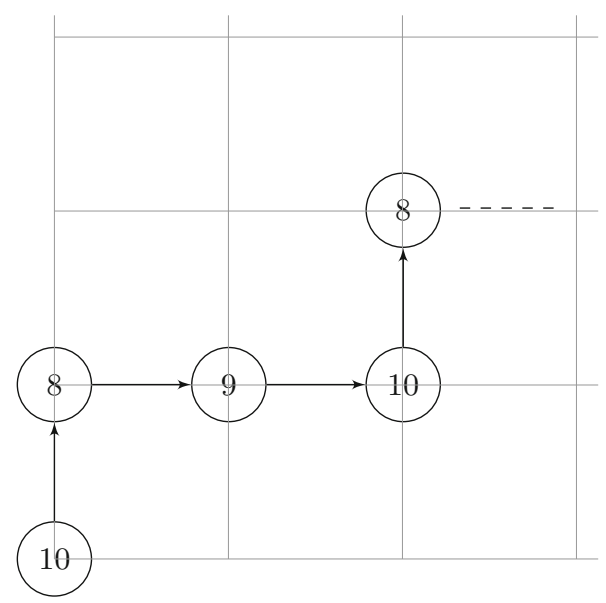

Fig. 2. Computations starting with $f(a)$ for $\mathcal{R}$ in Example 5

(a) If $x_{i+1}=x_{i}+1$, then $v_{i} \in \mathcal{D} \operatorname{Subterm}\left(\mathcal{R}, r_{i}\right)$ and $\alpha_{i} \in \operatorname{DRules}\left(\mathcal{R}, \ell_{i}\right)$.

(b) If $y_{i+1}=y_{i}+1$, then there is $j, 1 \leq j \leq n_{i}$ s.t. $v_{i} \in \mathcal{D} \operatorname{Subterm}\left(\mathcal{R}, s_{j}^{i}\right)$ and $\alpha_{i} \in \operatorname{Rules}_{C}\left(\mathcal{R}, \ell_{i}\right)$.

Example 5. Consider the following deterministic 3-CTRS $\mathcal{R}$ which is obtained from the 2-CTRS in Example 2 by a small change in rule (7) to yield (10):

$$
\begin{aligned}
g(a) & \rightarrow c(b) \\
b & \rightarrow f(a) \\
f(x) & \rightarrow y \Leftarrow g(x) \rightarrow c(y)
\end{aligned}
$$

Figure 2 shows the representation of computation starting from $f(a) \in \mathcal{T}_{\text {op- } \infty}$ according to Theorem 1 , where the coordinates $\left(x_{i}, y_{i}\right)$ have been left implicit.

Remark 2. The minimal sequence $f(a) \rightarrow_{(10)} b \rightarrow_{(9)} f(a) \rightarrow_{(10)} b \rightarrow \cdots$ is also possible for $\mathcal{R}$ in Example 5. This is because $\sigma(g(x)) \rightarrow^{*} \sigma(c(y))$ for rule (10) is satisfied without any reduction on $b$ if $\sigma(x)=a$ and $\sigma(y)=b$. The implicit assumption in the computation model of Proposition 1 is that only reachability conditions $\sigma\left(s_{i}\right) \rightarrow^{*} \sigma\left(t_{i}\right)$ that are free of any infinite computation are important to decide the application of a rule. This makes real sense in practice. And, of course, it is harmless for the correctness or completeness of our approach.

According to our discussion, the following definition establishes the subsets of rules that play a special role in computations starting from minimal terms.

Definition 3. The dependent usable rules for a $\operatorname{CTRS} \mathcal{R}$ and $t \in \mathcal{T}(\mathcal{F}, \mathcal{X})$ are:

$$
\mathcal{D U}(\mathcal{R}, t)=\operatorname{DRules}(\mathcal{R}, t) \cup \bigcup_{(l \rightarrow r \Leftarrow c) \in \operatorname{DRules}(\mathcal{R}, t)} \bigcup_{v \in \mathcal{D} \operatorname{Subterm}(\mathcal{R}, r)} \mathcal{D U}(\mathcal{R} \bullet, v)
$$


where $\mathcal{R}^{\bullet}=\mathcal{R}-\operatorname{DRules}(\mathcal{R}, t)$. The set of minimal usable rules of $\mathcal{R}$ for $t$ is:

$$
\mathcal{M U}(\mathcal{R}, t)=\operatorname{URules}(\mathcal{R}, t) \cup \bigcup_{(l \rightarrow r \Leftarrow c) \in D \operatorname{Rules}(\mathcal{R}, t)} \bigcup_{v \in \mathcal{D S u b t e r m}(\mathcal{R}, r)} \operatorname{M\mathcal {U}}\left(\mathcal{R}^{\bullet}, v\right) .
$$

Let $\overline{\mathcal{M U}}(\mathcal{R}, t)=\emptyset$ if $\mathcal{M U}(\mathcal{R}, t)$ is a TRS and $\overline{\mathcal{M U}}(\mathcal{R}, t)=\mathcal{M U}(\mathcal{R}, t)$ otherwise.

Example 6. For $\mathcal{R}$ in Example 1, $\mathcal{D U}(\mathcal{R}, h(x))=\mathcal{M U}(\mathcal{R}, h(x))=\emptyset ; \mathcal{D U}(\mathcal{R}, g(x))$ $=\emptyset$ but $\mathcal{M U}(\mathcal{R}, g(x))=\overline{\mathcal{M U}}(\mathcal{R}, g(x))=\{(4)\}$, and $\mathcal{D U}(\mathcal{R}, f(x, x, x))=$ $\mathcal{M U}(\mathcal{R}, f(x, x, x))=\{(3)\}$, but $\overline{\mathcal{M U}}(\mathcal{R}, f(x, x, x))=\emptyset$.

Example 7. For $\mathcal{R}$ in Example 5, DRules $(\mathcal{R}, f(a))=\emptyset$ (because the right-hand side $y$ in rule (10) defining $f$ is a variable), $\mathcal{D U}(\mathcal{R}, g(x))=\{(8),(9)\}$ and $\mathcal{M U}(\mathcal{R}, g(x))=\overline{\mathcal{M U}}(\mathcal{R}, g(x))=\mathcal{R}$.

The following result shows that an infinite computation starting from a minimal operationally nonterminating term can either start an infinite (horizontal) rewrite sequence (possibly as part of the evaluation of one of the conditions of a rule) or else climb infinitely many 'vertical' steps over the conditions in the rules.

Corollary 1. Let $\mathcal{R}$ be a deterministic 3-CTRS and $t \in \mathcal{T}_{\text {op- } \infty}$. Then, the sequence $\left\{\left(x_{i}, y_{i}, \alpha_{i}\right)\right\}_{i \geq 0}$ associated to $t$ according to Theorem 1 satisfies one of the following conditions. Either

1. There is $k \geq 0, \ell_{k} \rightarrow r_{k} \Leftarrow c_{k} \in \mathcal{R}$, and an infinite 'horizontal' sequence $\left\{\left(x_{i}, y_{k}, \alpha_{i}\right)\right\}_{i \geq k}$ such that for all $i \geq k, x_{i+1}=x_{i}+1$ and $\alpha_{i} \in$ $v_{k} \in \mathcal{D}$ Subterm $\left(\mathcal{R}, r_{k}\right)$ $\mathcal{D U}\left(\mathcal{R}, v_{k}\right)$, or

2. For each $i \in \mathbb{N}$ such that $y_{i}>0$ and $y_{i}=y_{i-1}+1$, there is $k_{i}>i$ such that $y_{k_{i}}=y_{i}+1$, and there is $j_{i}, 1 \leq j_{i} \leq n_{i}$ such that $\alpha_{k_{i}-1} \in$ $v_{i} \in \mathcal{D} \operatorname{Subterm}\left(\mathcal{R}, s_{i j_{i}}\right)$

part of the rule.

In the following, we use Dependency Pairs to capture the nontermination behavior of computations with CTRSs.

\section{2D Dependency Pairs for CTRSs}

Given a signature $\mathcal{F}$ and $f \in \mathcal{F}$, we let $f^{\sharp}$ (often just capitalized, e.g., $F$ ) be a fresh symbol associated to $f[1]$. Let $\mathcal{F}^{\sharp}=\left\{f^{\sharp} \mid f \in \mathcal{F}\right\}$. For $t=f\left(t_{1}, \ldots, t_{k}\right) \in$ $\mathcal{T}(\mathcal{F}, \mathcal{X})$, we write $t^{\sharp}$ to denote the marked term $f^{\sharp}\left(t_{1}, \ldots, t_{k}\right)$. Our Dependency Pairs for CTRSs are organized into two blocks. The horizontal block contains those pairs that correspond to rules issuing root steps in infinite rewrite sequences (Proposition 1, item 1):

$$
\mathrm{DP}_{H}(\mathcal{R})=\left\{\ell^{\sharp} \rightarrow v^{\sharp} \Leftarrow c \mid \ell \rightarrow r \Leftarrow c \in R, r \unrhd v, \ell \not v, \operatorname{DRules}(\mathcal{R}, v) \neq \emptyset\right\}
$$


Example 8. For $\mathcal{R}$ in Example 1, DP ${ }_{H}(\mathcal{R})=\{F(k(a), k(b), x) \rightarrow F(x, x, x)\}$. For $\mathcal{R}$ in Example 5 (and also for $\mathcal{R}$ in Example 2), $\mathrm{DP}_{H}(\mathcal{R})=\{G(a) \rightarrow B\}$.

The vertical block contains pairs for shifting the infinite computation to the conditions of the rules (Proposition 1, item 2):

$$
\begin{aligned}
\operatorname{DP}_{V}(\mathcal{R})=\left\{\ell^{\sharp} \rightarrow\right. & v^{\sharp} \Leftarrow \bigwedge_{j=1}^{k-1} s_{j} \rightarrow t_{j} \mid \ell \rightarrow r \Leftarrow \bigwedge_{i=1}^{n} s_{i} \rightarrow t_{i} \in R, \\
& \left.\exists k, 1 \leq k \leq n, s_{k} \unrhd v, \ell \triangleright v, \operatorname{URules}(\mathcal{R}, v) \neq \emptyset\right\} .
\end{aligned}
$$

Example 9. For $\mathcal{R}$ in Example 1, $\operatorname{DP}_{V}(\mathcal{R})=\emptyset$. For $\mathcal{R}$ in Example 2 and $\mathcal{R}$ in Example 5), $\operatorname{DP}_{V}(\mathcal{R})=\{F(x) \rightarrow G(x)\}$.

The subterms in the conditions of the rules that originate the pairs in $\operatorname{DP}_{V}(\mathcal{R})$ are collected in the following set, which we use below:

$\mathrm{V}_{C}(\mathcal{R})=\left\{v \mid \ell \rightarrow r \Leftarrow \bigwedge_{i=1}^{n} s_{i} \rightarrow t_{i} \in R, \exists k, 1 \leq k \leq n, s_{k} \unrhd v, \ell \not v, \operatorname{URules}(\mathcal{R}, v) \neq \emptyset\right\}$.

Example 10. For $\mathcal{R}$ in Example $1, \mathrm{~V}_{C}(\mathcal{R})=\emptyset$. For $\mathcal{R}$ in Example 2 and $\mathcal{R}$ in Example 5, $\mathrm{V}_{C}(\mathcal{R})=\{g(x)\}$.

We also have pairs to connect pairs in $\operatorname{DP}_{V}(\mathcal{R})$ (Corollary 1, item 1):

$$
\begin{gathered}
\operatorname{DP}_{V_{H}}(\mathcal{R})=\bigcup_{w \in \mathrm{V}_{C}(\mathcal{R})}\left\{\ell^{\sharp} \rightarrow v^{\sharp} \Leftarrow c \mid \ell \rightarrow r \Leftarrow c \in \overline{\mathcal{M U}}(\mathcal{R}, w),\right. \\
r \unrhd v, \ell \not v, \operatorname{URules}(\mathcal{R}, v) \neq \emptyset\} .
\end{gathered}
$$

Example 11. For $\mathcal{R}$ in Example 1, $\mathrm{DP}_{V H}(\mathcal{R})=\emptyset$. For $\mathcal{R}$ in Example 2 and $\mathcal{R}$ in Example 5, $\operatorname{DP}_{V H}(\mathcal{R})=\{G(a) \rightarrow B, B \rightarrow F(a)\}$.

Here is the definition of $2 D$-Dependency Pairs for a CTRS.

Definition 4 (2D-Dependency Pairs). The triple of 2D-dependency pairs (2D DPs) for the CTRS $\mathcal{R}$ is $\mathrm{DP}_{2 \mathrm{D}}(\mathcal{R})=\left(\mathrm{DP}_{H}(\mathcal{R}), \mathrm{DP}_{V}(\mathcal{R}), \mathrm{DP}_{V H}(\mathcal{R})\right)$.

Example 12. Consider the following 3-CTRS $\mathcal{R}$ in [14, Example 7.1.5]

$$
\begin{aligned}
\operatorname{less}(x, 0) & \rightarrow \text { false } \\
\operatorname{less}(0, \mathrm{~s}(x)) & \rightarrow \text { true } \\
\operatorname{less}(\mathrm{s}(x), \mathrm{s}(y)) & \rightarrow \operatorname{less}(x, y) \\
\operatorname{minus}(0, \mathrm{~s}(y)) & \rightarrow 0 \\
\operatorname{minus}(x, 0) & \rightarrow x \\
\operatorname{minus}(\mathrm{s}(x), \mathrm{s}(y)) & \rightarrow \operatorname{minus}(x, y) \\
\text { quotrem }(0, \mathrm{~s}(y)) & \rightarrow \operatorname{pair}(0,0) \\
\text { quotrem }(\mathrm{s}(x), \mathrm{s}(y)) & \rightarrow \operatorname{pair}(0, \mathrm{~s}(x)) \Leftarrow \operatorname{less}(x, y) \rightarrow \text { true } \\
\text { quotrem }(\mathrm{s}(x), \mathrm{s}(y)) & \rightarrow \operatorname{pair}(\mathrm{s}(q), r) \\
& \Leftarrow \operatorname{less}(x, y) \rightarrow \text { false, quotrem }(\operatorname{minus}(x, y), \mathrm{s}(y)) \rightarrow \operatorname{pair}(q, r)
\end{aligned}
$$


The set $\mathrm{DP}_{H}(\mathcal{R})$ consists of the rules:

$$
\begin{aligned}
\operatorname{LESS}(\mathrm{s}(x), \mathrm{s}(y)) & \rightarrow \operatorname{LESS}(x, y) \\
\operatorname{MINUS}(\mathrm{s}(x), \mathrm{s}(y)) & \rightarrow \operatorname{MinUS}(x, y)
\end{aligned}
$$

The set $\operatorname{DP}_{V}(\mathcal{R})$ consists of the rules:

$$
\begin{aligned}
& \text { QUOTREM }(\mathrm{s}(x), \mathrm{s}(y)) \rightarrow \operatorname{LESS}(x, y) \\
& \text { QUOTREM }(\mathrm{s}(x), \mathrm{s}(y)) \rightarrow \operatorname{QUOTREM}(\operatorname{minus}(x, y), \mathrm{s}(y)) \Leftarrow \text { less }(x, y) \rightarrow \text { false } \\
& \text { QUOTREM }(\mathrm{s}(x), \mathrm{s}(y)) \rightarrow \operatorname{MinUS}(x, y) \Leftarrow \text { less }(x, y) \rightarrow \text { false }
\end{aligned}
$$

Finally, $\mathrm{DP}_{V H}(\mathcal{R})=\emptyset$.

\subsection{Characterizing Operational Termination of CTRSs Using 2D DPs}

An essential property of the dependency pair method is that it provides a characterization of termination of a TRS $\mathcal{R}$ as the absence of infinite (minimal) chains of dependency pairs $[1,8]$. As we prove below, this is also true for deterministic 3-CTRSs when 2D DPs are considered. First, we have to introduce a suitable notion of chain that can be used with 2D DPs.

Definition 5 (Chain of Pairs - Minimal Chain). Let $\mathcal{P}, \mathcal{Q}, \mathcal{R}$ be CTRSs. $A(\mathcal{P}, \mathcal{Q}, \mathcal{R})$-chain is a finite or infinite sequence of pairs $u_{i} \rightarrow v_{i} \Leftarrow \bigwedge_{j=1}^{n_{i}} s_{i j} \rightarrow$ $t_{i j} \in \mathcal{P}$, together with a substitution $\sigma$ satisfying that, for all $i \geq 1$,

1. $\sigma\left(s_{i j}\right) \rightarrow_{\mathcal{R}}^{*} \sigma\left(t_{i j}\right)$ for all $j, 1 \leq j \leq n_{i}$ and

2. $\sigma\left(v_{i}\right)\left(\rightarrow_{\mathcal{R}}^{*} \circ \stackrel{\Lambda}{\longrightarrow} \overline{\overline{\mathcal{Q}}}\right)^{*} \sigma\left(u_{i+1}\right)$, where given a rule $\ell \rightarrow r \Leftarrow \bigwedge_{j=1}^{n} s_{j} \rightarrow t_{j} \in \mathcal{Q}$, we write $s \stackrel{\Lambda}{\longrightarrow} \overline{\overline{\mathcal{Q}}} t$ if either $s=t$ or there is a substitution $\theta$ such that $s=\theta(\ell)$, $t=\theta(r)$ and $\theta\left(s_{i}\right) \rightarrow_{\mathcal{R}}^{*} \theta\left(t_{i}\right)$ for all $j, 1 \leq j \leq n$ (note that the satisfaction of reachability constraints involves rewritings with $\mathcal{R})$.

As usual, we assume that different occurrences of pairs do not share any variable (renaming substitutions are used if necessary). $A(\mathcal{P}, \mathcal{Q}, \mathcal{R})$-chain is called minimal if for all $i \geq 1, \sigma\left(v_{i}\right)$ is $\mathcal{R}$-operationally terminating.

Remark 3. Note that, if $\mathcal{P}$ and $\mathcal{R}$ are TRSs (without conditional rules) and $\mathcal{Q}=\emptyset$, Definition 5 specializes to the standard definition of chain of pairs in the Dependency Pair Framework for TRSs [8, Definition 3].

We now provide a new characterization of operational termination of CTRSs.

Theorem 2 (Operational Termination of CTRSs). A deterministic 3-CTRS $\mathcal{R}$ is operationally terminating if and only if there is no infinite (minimal) $\left(\mathrm{DP}_{H}(\mathcal{R}), \emptyset, \mathcal{R}\right)$-chain and there is no infinite $\left(\right.$ minimal) $\left(\operatorname{DP}_{V}(\mathcal{R}), \operatorname{DP}_{V H}(\mathcal{R}), \mathcal{R}\right)$ chain. 
Example 13. Consider again the $\mathcal{R}$ in Examples 2 and 5 and $\operatorname{DP}_{V}(\mathcal{R})$ and $\operatorname{DP}_{V H}(\mathcal{R})$ (that coincide for both CTRSs) as given in Examples 9 and 11. There is an infinite $\left(\operatorname{DP}_{V}(\mathcal{R}), \operatorname{DP}_{V H}(\mathcal{R}), \mathcal{R}\right)$-chain:

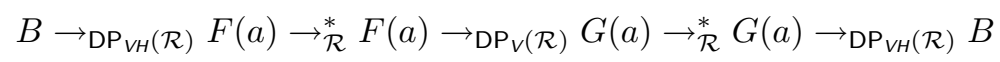

witnessing that both CTRSs are not operationally terminating.

For the sake of brevity, in the following we often call $H$-chains to the $\left(\mathrm{DP}_{H}(\mathcal{R}), \emptyset, \mathcal{R}\right)$-chains. And we call $V$-chains to the $\left(\operatorname{DP}_{V}(\mathcal{R}), \mathrm{DP}_{V H}(\mathcal{R}), \mathcal{R}\right)$ chains. The following result, involving chains of a simpler type (closer to the usual ones, where pairs are connected by rewritings with $\mathcal{R}$ only, see Remark 3 ), also characterizes operational termination of deterministic 3-CTRSs.

Theorem 3 (Operational Termination of CTRSs II). A deterministic 3$C T R S \mathcal{R}$ is operationally terminating if and only if there is no infinite (minimal) $\left(\mathrm{DP}_{H}(\mathcal{R}) \cup \operatorname{DP}_{V}(\mathcal{R}) \cup \operatorname{DP}_{V H}(\mathcal{R}), \emptyset, \mathcal{R}\right)$-chain.

In the following section, though, we further motivate the explicit and independent use of the $H$-chains and $V$-chains to prove termination properties of CTRSs.

\subsection{Termination of 2-CTRSs}

The existence of infinite $H$-chains witnesses nontermination of deterministic 3CTRSs, i.e., the absence of infinite rewrite sequences.

Theorem 4 (Non-termination of CTRSs). Let $\mathcal{R}$ be a deterministic 3CTRS. If there is an infinite $\left(\mathrm{DP}_{H}(\mathcal{R}), \emptyset, \mathcal{R}\right)$-chain, then $\mathcal{R}$ is not terminating.

The CTRS $\mathcal{R}$ in Example 5 shows that Theorem 4 provides a sufficient but not necessary criterion for termination of CTRSs.

Example 14. For $\mathcal{R}$ in Example 5, we have $\mathrm{DP}_{H}(\mathcal{R})=\{G(a) \rightarrow B\}$ (Example $8)$. There is no infinite $H$-chain. However, $\mathcal{R}$ is not terminating (see Remark 2).

However, the following result holds:

Theorem 5 (Termination of 2-CTRSs.) A 2-CTRS $\mathcal{R}$ is terminating if and only if there is no infinite minimal $\left(\mathrm{DP}_{H}(\mathcal{R}), \emptyset, \mathcal{R}\right)$-chain.

Example 15. For the deterministic 2-CTRS $\mathcal{R}$ in Example 2, $\mathrm{DP}_{H}(\mathcal{R})=\{G(a) \rightarrow$ $B\}$ and there is no infinite $H$-chain. By Theorem $5, \mathcal{R}$ is terminating.

Therefore, for CTRSs with extra variables in the right-hand sides of conditional rules, the vertical and horizontal dimensions of operational termination are not completely independent. Theorem 5 suggests the following.

Definition 6. ( $V$-termination of CTRSs). A CTRS $\mathcal{R}$ is $V$-terminating if there is no infinite $\left(\operatorname{DP}_{V}(\mathcal{R}), \operatorname{DP}_{V H}(\mathcal{R}), \mathcal{R}\right)$-chain.

As a consequence of Theorems 2 and 5, we have the following.

Corollary 2. A deterministic 2-CTRS is operationally terminating if and only if it is terminating and $V$-terminating. 


\section{Mechanizing Proofs of Operational Termination with 2D DPs}

In the following, we speak of $(\mathcal{P}, \mathcal{Q}, \mathcal{R},($ ctrs, $\gamma))$-chains, for $\gamma=$ a (or $\gamma=\mathrm{m})$ if arbitrary (resp. only minimal) chains are considered. Similarly, according to Remark 3 , we speak of $(\mathcal{P}, \mathcal{Q}, \mathcal{R},(\operatorname{trs}, \gamma))$-chains if $\mathcal{P}$ and $\mathcal{R}$ are TRSs and $\mathcal{Q}=\emptyset$.

Definition 7 (CTRS Problem). A CTRS problem $\tau$ is a tuple $\tau=(\mathcal{P}, \mathcal{Q}, \mathcal{R}, e)$, where $\mathcal{P}, \mathcal{Q}$ and $\mathcal{R}$ are $C T R S s$, and $e \in\{\mathrm{ctrs}, \operatorname{trs}\} \times\{\mathrm{a}, \mathrm{m}\}$ is a flag. The CTRS problem $\tau$ is finite if there is no infinite minimal $(\mathcal{P}, \mathcal{Q}, \mathcal{R}, e)$-chain. The CTRS problem $\tau$ is infinite if $\mathcal{R}$ is non-operationally terminating or there is an infinite minimal $(\mathcal{P}, \mathcal{Q}, \mathcal{R}, e)$-chain.

Definition 8 (CTRS Processor). A CTRS processor P is a mapping from CTRS problems into sets of CTRS problems. Alternatively, it can also return "no". A CTRS processor $\mathrm{P}$ is

- sound if for all CTRS problems $\tau$, we have that $\tau$ is finite whenever $\mathrm{P}(\tau) \neq$ no and all CTRS problems in $\mathrm{P}(\tau)$ are finite.

- complete if for all CTRS problems $\tau$, we have that $\tau$ is infinite whenever $\mathrm{P}(\tau)=$ no or when $\mathrm{P}(\tau)$ contains an infinite CTRS problem.

A (sound) processor transforms CTRS problems into (hopefully) simpler ones, in such a way that the existence of an infinite chain in the original CTRS problem implies the existence of an infinite chain in the transformed one. Here, 'simpler' usually means that fewer pairs are involved. Soundness is essential for proving operational termination; completeness for proving non-operational termination.

Processors are used in a divide and conquer scheme to incrementally simplify the original CTRS problem as much as possible, possibly decomposing it into (a tree of) smaller pieces which are independently treated in the same way. The trivial case comes when the set of pairs $\mathcal{P}$ becomes empty. Then, no infinite chain is possible, and the CTRS problem is finite. Such positive answer is propagated upwards in the decision tree. In some cases, a witness of an infinite chain is obtained; then a negative answer "no" can be provided and propagated upwards.

Theorem 6 (2D DP Framework). Let $\mathcal{R}$ be a deterministic 3-CTRS. We construct two trees whose nodes are labeled with CTRS problems $\tau$ or "yes" or "no". The roots are $\tau_{H}=\left(\operatorname{DP}_{H}(\mathcal{R}), \emptyset, \mathcal{R},(\operatorname{ctrs}, \gamma)\right)$ and $\tau_{V}=\left(\operatorname{DP} V(\mathcal{R}), \operatorname{DP}_{V H}(\mathcal{R})\right.$, $\mathcal{R},(\mathrm{ctrs}, \gamma)$ ), respectively (for $\gamma \in\{\mathrm{a}, \mathrm{m}\}$ ). For every node which is a CTRS problem $\tau$, there is a sound processor $\mathrm{P}$ satisfying one of the following conditions:

1. $\mathrm{P}(\tau)=$ no and the node has just one child that is labeled with "no".

2. $\mathrm{P}(\tau)=\emptyset$ and the node has just one child that is labeled with "yes".

3. $\mathrm{P}(\tau) \neq$ no, $\mathrm{P}(\tau) \neq \emptyset$, and the children of the node are labeled with the CTRS problems in $\mathrm{P}(\tau)$. 
If all leaves of both trees are labeled with "yes", then $\mathcal{R}$ is operationally terminating. If a leaf is labeled with "no" in some of the trees and all processors used on the path from the root to this leaf are complete, then $\mathcal{R}$ is operationally nonterminating.

Remark 4. By Theorem 3, an alternative to the twofold proof starting from an $H$-problem and a $V$-problem is to start the proof of operational termination of $\mathcal{R}$ from a single CTRS problem $\left(\mathrm{DP}_{H}(\mathcal{R}) \cup \operatorname{DP}_{V}(\mathcal{R}) \cup \mathrm{DP}_{V H}(\mathcal{R}), \emptyset, \mathcal{R},(\right.$ ctrs,$\left.\gamma)\right)$.

Remark 5. In order to prove (or disprove) termination of a deterministic CTRS $\mathcal{R}$, we would use Theorem 6 with a single problem: $\tau_{H}=\left(\mathrm{DP}_{H}(\mathcal{R}), \emptyset, \mathcal{R}, e\right)$. The procedure is analogous and the conclusion of a positive analysis (i.e., "yes" in all leaves of the tree) is termination of $\mathcal{R}$ (if it is a 2-CTRS). Similarly, a leaf labeled with "no" witnesses nontermination of $\mathcal{R}$ (if it is a 3 -CTRS).

\section{Processors for the 2D DP Framework}

The first processor moves rules from $\mathcal{Q}$ to $\mathcal{P}$ in CTRS problems.

Theorem 7 (Moving $\mathcal{Q}$-rules). Let $\mathcal{P}, \mathcal{Q}$, and $\mathcal{R}$ be TRSs. Then,

$$
\mathrm{P}_{\mathrm{Q} 2 \mathrm{P}}(\mathcal{P}, \mathcal{Q}, \mathcal{R},(\text { ctrs }, \gamma))=\{(\mathcal{P} \cup \mathcal{Q}, \emptyset, \mathcal{R},(\text { ctrs }, \mathrm{a}))\}
$$

is a sound processor.

In general, $\mathrm{P}_{\mathrm{Q} 2 \mathrm{P}}$ is not complete nor preserves minimality.

Example 16. Let $\mathcal{P}=\{a \rightarrow b, c \rightarrow a\}, \mathcal{Q}=\{b \rightarrow c\}$, and $\mathcal{R}=\{c \rightarrow c\}$. There is an infinite $(\mathcal{P}, \mathcal{Q}, \mathcal{R})$-chain $\Gamma: a \rightarrow b, c \rightarrow a, a \rightarrow b, c \rightarrow a, \ldots$ due to $b \rightarrow_{\mathcal{Q}} c$. Note that $\Gamma$ is minimal because $b$ and $a$ are $\mathcal{R}$-terminating. However, $\Gamma$ requires the use of the (only) pair in $\mathcal{Q}$ to become an infinite $(\mathcal{P} \cup \mathcal{Q}, \emptyset, \mathcal{R})$-chain

$$
a \rightarrow b, b \rightarrow c, c \rightarrow a, a \rightarrow b, b \rightarrow c, c \rightarrow a, \ldots
$$

which is, however, not minimal now because $c$ is not $\mathcal{R}$-terminating.

The following processor transfers any proof of finiteness of 2D DP problems to the DP Framework for TRSs. In this way, all existing processors for the DP Framework are now available for the 2D DP framework.

Theorem 8 (Shift to DP-Framework). Let $\mathcal{P}$ and $\mathcal{R}$ be TRSs. Then,

$$
\mathrm{P}_{T R S}(\mathcal{P}, \emptyset, \mathcal{R},(\operatorname{ctrs}, \gamma))=\{(\mathcal{P}, \emptyset, \mathcal{R},(\operatorname{trs}, \gamma))\}
$$

is a sound and complete processor. 


\subsection{Graph of a CTRS Problem}

Given a CTRS problem $(\mathcal{P}, \mathcal{Q}, \mathcal{R}, e)$, we provide a notion of graph that is able to represent all infinite (minimal) chains of pairs as given in Definition 5.

Definition 9 (Graph of a CTRS Problem). Let $\mathcal{P}, \mathcal{Q}$ and $\mathcal{R}$ be CTRSs. The CTRS-graph $\mathrm{G}(\mathcal{P}, \mathcal{Q}, \mathcal{R}, e)$ where $e=(\mathrm{ctrs}, \gamma)$ and $\gamma \in\{\mathrm{a}, \mathrm{m}\}$ has $\mathcal{P}$ as the set of nodes. Given $\alpha: u \rightarrow v \Leftarrow c, \alpha^{\prime}: u^{\prime} \rightarrow v^{\prime} \Leftarrow c^{\prime} \in \mathcal{P}$, there is an arc from $\alpha$ to $\alpha^{\prime}$ if $\alpha, \alpha^{\prime}$ is a minimal $(\mathcal{P}, \mathcal{Q}, \mathcal{R}, e)$-chain for some substitution $\sigma$.

In general, CTRS graphs are not computable due to the reachability conditions $\sigma(v)\left(\rightarrow_{\mathcal{R}}^{*} \circ \stackrel{\Lambda}{\longrightarrow} \overline{\mathcal{Q}}\right)^{*} \sigma\left(u^{\prime}\right)$ (for $u \rightarrow v \Leftarrow c \in \mathcal{P}$ ). Since the reachability problem for (conditional) rewriting is undecidable, we approximate it. Following [9], we approximate the CTRS-dependency graph as follows. Let $\operatorname{TCAP}_{\mathcal{R}}$ be:

$$
\begin{aligned}
& \operatorname{TCAP}_{\mathcal{R}}(x)=y \text { if } x \text { is a variable, and } \\
& \operatorname{TCAP}_{\mathcal{R}}\left(f\left(t_{1}, \ldots, t_{k}\right)\right)= \begin{cases}f\left(\left[t_{1}\right], \ldots,\left[t_{k}\right]\right) & \text { if } f\left(\left[t_{1}\right], \ldots,\left[t_{k}\right]\right) \text { does not unify } \\
y & \text { with } \ell \text { for any } \ell \rightarrow r \Leftarrow c \text { in } \mathcal{R}\end{cases} \\
& \text { otherwise }
\end{aligned}
$$

where $y$ is a new, fresh variable that has not yet been used, and given a term $s,[s]=\operatorname{TCAP}_{\mathcal{R}}(s)$. We assume that $\ell$ shares no variable with $f\left(\left[t_{1}\right], \ldots,\left[t_{k}\right]\right)$ (rename if necessary). With $\operatorname{TCAP}_{\mathcal{R}}$ we approximate reachability problems as unification. According to Definitions 5 and 9, we have the following.

Definition 10 (Estimated connection). Let $\mathcal{Q}$ and $\mathcal{R}$ be CTRSs, $\theta$ be a substitution, and $\alpha: u \rightarrow v \Leftarrow c$ to $\alpha^{\prime}: u^{\prime} \rightarrow v^{\prime} \Leftarrow c^{\prime}$ be two conditional rules. There is a $(\mathcal{Q}, \mathcal{R}, \theta)$-connection from $\alpha$ to $\alpha^{\prime}$ if

1. $\operatorname{TCAP}_{\mathcal{R}}(\theta(v))$ and $u^{\prime}$ unify, or

2. $\operatorname{TCAP}_{\mathcal{R}}(\theta(v))$ and $u^{\prime \prime}$ unify with mgu $\theta^{\prime}$ for some $\alpha^{\prime \prime}: u^{\prime \prime} \rightarrow v^{\prime \prime} \Leftarrow c^{\prime \prime} \in \mathcal{Q}$ and there is a $\left(\mathcal{Q}-\left\{\alpha^{\prime \prime}\right\}, \mathcal{R}, \theta^{\prime}\right)$-connection from $\alpha^{\prime \prime}$ to $\alpha^{\prime}$.

Definition 11 (Estimated Graph). Let $\mathcal{P}, \mathcal{Q}$ and $\mathcal{R}$ be CTRSs. The estimated $C T R S$-graph $\mathrm{EG}(\mathcal{P}, \mathcal{Q}, \mathcal{R}, e)$ has $\mathcal{P}$ as the set of nodes. There is an arc from $\alpha$ to $\alpha^{\prime}$ if there is a $(\mathcal{Q}, \mathcal{R}, \epsilon)$-connection from $\alpha$ to $\alpha^{\prime}$.

Remark 6. If $\mathcal{Q}=\emptyset$ and $\mathcal{P}, \mathcal{R}$ are TRSs, Definitions 9 and 11 specialize to the standard ones for TRSs [8, Definition 7] (and [9, Definition 12]).

The following processor decomposes a CTRS problem $(\mathcal{P}, \mathcal{Q}, \mathcal{R}, e)$ with graph $\mathrm{G}(\mathcal{P}, \mathcal{Q}, \mathcal{R}, e)$ according to the strongly connected components (SCCs) of the graph, i.e., cycles in $\mathrm{G}(\mathcal{P}, \mathcal{Q}, \mathcal{R}, e)$ that are not contained in any other cycle.

Theorem 9 (SCC Processor). Let $\mathcal{P}, \mathcal{Q}$ and $\mathcal{R}$ be CTRSs. Then,

$$
\mathrm{P}_{S C C}(\mathcal{P}, \mathcal{Q}, \mathcal{R}, e)=\left\{\left(\mathcal{P}^{\prime}, \mathcal{Q}, \mathcal{R}, e\right) \mid \mathcal{P}^{\prime} \subseteq \mathcal{P} \text { is an } \operatorname{SCC} \text { in } \mathrm{G}(\mathcal{P}, \mathcal{Q}, \mathcal{R}, e)\right\}
$$

is a sound and complete processor. 
With $\mathrm{P}_{S C C}$, we can separately work with the strongly connected components of $\mathrm{G}(\mathcal{P}, \mathcal{Q}, \mathcal{R}, e)$, disregarding other parts of the graph.

Example 1\%. For $\mathcal{R}$ in Example 12, $\tau_{H}=\left(\mathrm{DP}_{H}(\mathcal{R}), \emptyset, \mathcal{R}, e\right)$ and $\tau_{V}=\left(\operatorname{DP}_{V}(\mathcal{R})\right.$, $\left.\mathrm{DP}_{V H}(\mathcal{R}), \mathcal{R}, e\right) ; \mathrm{EG}\left(\tau_{H}\right)$ and $\mathrm{EG}\left(\tau_{V}\right)$ are:
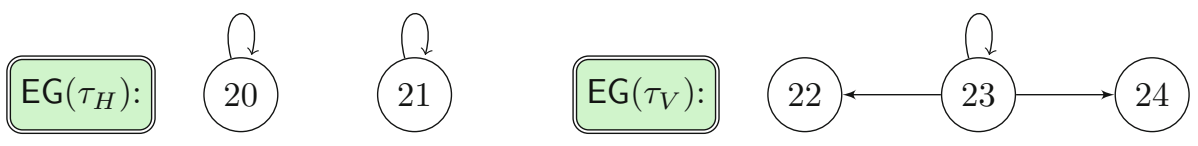

We have $\mathrm{P}_{S C C}\left(\tau_{H}\right)=\left\{\tau_{H 1}, \tau_{H 2}\right\}$, where $\tau_{H 1}=(\{(20)\}, \emptyset, \mathcal{R}, e)$ and $\tau_{H 2}=$ $(\{(21)\}, \emptyset, \mathcal{R}, e)$. For $\tau_{V}$ we get $\mathrm{P}_{S C C}\left(\tau_{V}\right)=\left\{\tau_{V 1}\right\}$, where $\tau_{V 1}=(\{(23)\}, \emptyset, \mathcal{R}, e)$.

\subsection{Use of Orderings and Argument Filterings}

A CTRS problem $(\mathcal{P}, \mathcal{Q}, \mathcal{R}, e)$ can be simplified by removing rules with a decrease with respect to a well-founded relation $\sqsupset$. In order to be more precise, in the following we say that a relation $S$ is compatible with $R$ if $S \circ R \subseteq R$ or $R \circ S \subseteq R$.

Definition 12 (Removal Triple). A removal triple $(\gtrsim, \succeq, \sqsupset)$ consists of relations $\gtrsim, \succeq, \sqsupset$ on terms such that $\sqsupset$ is well-founded; for all $R \in\{\gtrsim, \succeq\}, R$ is compatible with $\sqsupset$; and $\gtrsim \circ \succeq \subseteq \gtrsim$ or $\gtrsim \circ \succeq \subseteq \succeq$.

An argument filtering $\pi$ for a signature $\mathcal{F}$ is a mapping that assigns to each $k$-ary function symbol $f \in \mathcal{F}$ an argument position $i \in\{1, \ldots, k\}$ or a (possibly empty) list $\left[i_{1}, \ldots, i_{m}\right]$ of argument positions $1 \leq i_{1}<\cdots<i_{m} \leq k$ [11]. The trivial argument filtering $\pi_{\top}(f)=[1, \ldots, k]$ (for each $k$-ary symbol $f \in \mathcal{F}$ ) does nothing. The signature $\mathcal{F}_{\pi}$ of symbols with filtered arguments consists of all function symbols $f \in \mathcal{F}$ such that $\pi(f)=\left[i_{1}, \ldots, i_{m}\right]$; the arity of $f$ in $\mathcal{F}_{\pi}$ is $m$ but we do not change the name. An argument filtering $\pi$ induces a mapping from $\mathcal{T}(\mathcal{F}, \mathcal{X})$ to $\mathcal{T}\left(\mathcal{F}_{\pi}, \mathcal{X}\right)$, also denoted by $\pi$, which removes subterms:

$$
\pi(t)= \begin{cases}t & \text { if } t \text { is a variable } \\ \pi\left(t_{i}\right) & \text { if } t=f\left(t_{1}, \ldots, t_{k}\right) \text { and } \pi(f)=i \\ f\left(\pi\left(t_{i_{1}}\right), \ldots, \pi\left(t_{i_{m}}\right)\right) & \text { if } t=f\left(t_{1}, \ldots, t_{k}\right) \text { and } \pi(f)=\left[i_{1}, \ldots, i_{m}\right]\end{cases}
$$

And if $R$ is a relation on terms, we let $\pi(R)=\{(\pi(s), \pi(t)) \mid(s, t) \in R\}$. Argument filterings provide a simple way to remove parts of the syntactic structure of a rule. In this way, we obtain simpler rules that are easier to compare. In the following, given (possibly empty) set of rules $\mathcal{R}, \mathcal{S}$ and a rule $\alpha: \ell \rightarrow r \Leftarrow c$, we define the (possible) replacement of $\alpha$ in $\mathcal{R}$ by the rules $\mathcal{S}$ as follows:

$$
\mathcal{R}[\mathcal{S}]_{\alpha}=\left\{\begin{array}{lr}
(\mathcal{R}-\{\alpha\}) \cup \mathcal{S} \text { if } \alpha \in \mathcal{R} \\
\mathcal{R} & \text { otherwise }
\end{array}\right.
$$


Theorem 10 (Removal Triple Processor). Let $\mathcal{P}, \mathcal{Q}$, and $\mathcal{R}$ be CTRSs, $\pi$ be an argument filtering and $(\gtrsim, \succeq, \sqsupset)$ be a removal triple such that $\pi\left(\rightarrow_{\mathcal{R}}^{*}\right) \subseteq \gtrsim$ and for all $\ell \rightarrow r \Leftarrow c \in \mathcal{P} \cup \mathcal{Q}$ and substitutions $\sigma$, if for all $s \rightarrow t \in c$, $\sigma(s) \rightarrow_{\mathcal{R}}^{*} \sigma(t)$ holds, then $\pi(\sigma(\ell)) \bowtie \pi(\sigma(r))$ holds for some $\bowtie \in\{\gtrsim, \succeq, \sqsupset\}$. Let $\alpha: u \rightarrow v \Leftarrow c \in \mathcal{P} \cup \mathcal{Q}$ be such that, for all substitutions $\sigma$, if for all $s \rightarrow t \in c$, $\sigma(s) \rightarrow_{\mathcal{R}}^{*} \sigma(t)$ holds, then $\pi(\sigma(u)) \sqsupset \pi(\sigma(v))$ holds. Then,

$$
\mathrm{P}_{R T}(\mathcal{P}, \mathcal{Q}, \mathcal{R}, e)=\left\{\left(\mathcal{P}[\emptyset]_{\alpha}, \mathcal{Q}[\emptyset]_{\alpha}, \mathcal{R}, e\right)\right\}
$$

is a sound and complete processor.

Example 18. For $\tau_{H 1}, \tau_{H 2}$ and $\tau_{V 1}$ in Example 17, we apply $\mathrm{P}_{R T}$ to those problems with $\pi_{\top}$ (which we do not make explicit here, as it does nothing) and using the same removal triple $(\geq, \geq,>)$ induced by the polynomial interpretation

$$
\begin{aligned}
& \text { [false }]=0 \\
& \text { [true }]=0 \\
& {[\text { less }](x)=0 \quad[\text { minus }](x, y)=x} \\
& {[\operatorname{LESS}](x, y)=x \quad[\operatorname{MINUS}](x, y)=x} \\
& {[0]=0} \\
& {[\text { pair }](x, y)=0} \\
& {[\mathbf{s}](x)=x+1} \\
& \text { [quotrem] }(x, y)=0
\end{aligned}
$$

over the naturals $\mathbb{N}$ by $s \geq t$ if $[s] \geq[t]$ and $s>t$ if $[s]>[t]$. We have:

$$
\begin{aligned}
& {[\operatorname{less}(x, 0)]=0 \geq 0=[\text { false }]} \\
& {[\operatorname{less}(0, \mathrm{~s}(x))]=0 \geq 0=[\operatorname{true}]} \\
& {[\operatorname{less}(\mathrm{s}(x), \mathrm{s}(y))]=0 \geq 0=[\operatorname{less}(x, y)]} \\
& {[\operatorname{minus}(0, \mathrm{~s}(y))]=0 \geq 0=[0]} \\
& {[\operatorname{minus}(x, 0)]=x \geq x=[x]} \\
& {[\operatorname{minus}(\mathrm{s}(x), \mathrm{s}(y))]=x+1 \geq x=[\operatorname{minus}(x, y)]} \\
& {[\text { quotrem }(0, \mathrm{~s}(y))]=0 \geq 0=[\operatorname{pair}(0,0)]} \\
& \text { [quotrem }(\mathrm{s}(x), \mathrm{s}(y))]=0 \geq 0=[\operatorname{pair}(0, \mathrm{~s}(x))] \\
& \text { [quotrem }(\mathrm{s}(x), \mathrm{s}(y))]=0 \geq 0=[\operatorname{pair}(\mathrm{s}(q), r)] \\
& {[\operatorname{LESS}(\mathrm{s}(x), \mathrm{s}(y))]=x+1>x=[\operatorname{LESS}(x, y)]} \\
& {[\operatorname{MinUS}(\mathrm{s}(x), \mathrm{s}(y))]=x+1>x=[\operatorname{MinUS}(x, y)]} \\
& {[\mathrm{QUOTREM}(\mathrm{s}(x), \mathrm{s}(y))]=x+1>x=[\operatorname{QUOTREM}(\operatorname{minus}(x, y), \mathrm{s}(y))]}
\end{aligned}
$$

Since $\geq$ is monotonic, stable, reflexive and transitive, the first nine inequalities prove $\rightarrow_{\mathcal{R}}^{*} \subseteq \geq$ (we do not really need to pay attention to the conditional part of the rules). Similarly, since $\sqsupset$ is stable, the last three strict inequalities prove $\sigma(u) \sqsupset \sigma(v)$ for all $u \rightarrow v \Leftarrow c \in \mathcal{P}$ (in the corresponding CTRS problem) and substitution $\sigma$, again without paying attention to the conditional part of the rules. This proves $\tau_{H 1}, \tau_{H 2}$ and $\tau_{V 1}$ finite, and $\mathcal{R}$ operationally terminating.

Theorem 11 (Unsatisfiable Rules). Let $\mathcal{P}, \mathcal{Q}$, and $\mathcal{R}$ be CTRSs, $\pi$ be an argument filtering, $\gtrsim$ and $\sqsupset$ be relations on terms such that $\gtrsim$ is compatible with $\sqsupset, \pi\left(\rightarrow_{\mathcal{R}}^{*}\right) \subseteq \gtrsim$, and $\sqsupset$ is well-founded. Let $\alpha: \ell \rightarrow r \Leftarrow c \in \mathcal{P} \cup \mathcal{Q} \cup \mathcal{R}$ and $s_{i} \rightarrow t_{i} \in c$ be such that for all substitutions $\sigma, \pi\left(\sigma\left(t_{i}\right)\right) \sqsupset \pi\left(\sigma\left(s_{i}\right)\right)$ holds. Then,

$$
\mathrm{P}_{U R}(\mathcal{P}, \mathcal{Q}, \mathcal{R}, e)=\left\{\left(\mathcal{P}[\emptyset]_{\alpha}, \mathcal{Q}[\emptyset]_{\alpha}, \mathcal{R}[\emptyset]_{\alpha}, e\right)\right\}
$$

is a sound and (if $\alpha \notin \mathcal{R}$ or $e=(\rho, \mathrm{a})$ ) complete processor. 
Example 19. For $\mathcal{R}$ in Example 1, $\mathrm{DP}_{H}(\mathcal{R})=\{F(k(a), k(b), x) \rightarrow F(x, x, x)\}$, and $\operatorname{DP}_{V}(\mathcal{R})=\operatorname{DP}_{V H}(\mathcal{R})=\emptyset$. For $\tau_{H}=\left(\operatorname{DP}_{H}(\mathcal{R}), \emptyset, \mathcal{R},(\right.$ ctrs, m $\left.)\right)$, we use

$$
[a]=[b]=[c](x)=[g](x)=[h](x)=[k](x)=[f](x, y, z)=0 \text { and }[d]=1
$$

to generate $\geq$ and easily show (as in Example 18) that $\rightarrow_{\mathcal{R}}^{*} \subseteq \geq$. Since $[h(x)]=$ 0 and $[d]=1$, we have $[d]>[h(x)]$. With $\mathrm{P}_{U R}$, we remove (4) from $\mathcal{R}$ to obtain $\tau_{H 1}=\left(\mathrm{DP}_{H}(\mathcal{R}), \emptyset, \mathcal{R}-\{(4)\},(\right.$ ctrs, m) $)$ that satisfies the conditions for a shift with $\mathrm{P}_{T R S}$ to a DP problem $\tau_{\text {trs }}=\left(\mathrm{DP}_{H}(\mathcal{R}), \emptyset, \mathcal{R}-\{(4)\},(\right.$ trs, $\left.\mathrm{m})\right)$ that can then be solved by using any processor for TRSs. For instance, the forward instantiation processor [8, Definition 28] can be used to prove finiteness of $\tau_{\text {trs }}$.

Theorem 12 (Unsatisfiable Rules II). Let $\mathcal{P}, \mathcal{Q}$, and $\mathcal{R}$ be $C T R S s, \pi$ be an argument filtering, $\gtrsim$ and $\sqsupset$ be relations on terms such that $\gtrsim$ is compatible with $\sqsupset$, $\sqsupset$ is well-founded, and $\pi(\rightarrow \mathcal{R}) \subseteq \neg$. Let $\alpha: \ell \rightarrow r \Leftarrow c \in \mathcal{P} \cup \mathcal{Q} \cup \mathcal{R}$ and $s_{i} \rightarrow t_{i} \in c$ be such that $\pi\left(s_{i}\right)$ and $\pi\left(t_{i}\right)$ do not unify and for all substitutions $\sigma$, $\pi\left(\sigma\left(t_{i}\right)\right) \gtrsim \pi\left(\sigma\left(s_{i}\right)\right)$ holds. Then,

$$
\mathrm{P}_{U R}(\mathcal{P}, \mathcal{Q}, \mathcal{R}, e)=\left\{\left(\mathcal{P}[\emptyset]_{\alpha}, \mathcal{Q}[\emptyset]_{\alpha}, \mathcal{R}[\emptyset]_{\alpha}, e\right)\right\}
$$

is a sound and (if $\alpha \notin \mathcal{R}$ or $e=(\rho, \mathrm{a})$ ) complete processor.

Example 20. Consider the following CTRS $\mathcal{R}[6$, p. 46]:

$$
a \rightarrow b \quad f(a) \rightarrow b \quad g(x) \rightarrow g(a) \Leftarrow f(x) \rightarrow x
$$

$\mathrm{DP}_{H}(\mathcal{R})$ consists of a single rule: $G(x) \rightarrow G(a) \Leftarrow f(x) \rightarrow x$ and $\operatorname{DP}_{V}(\mathcal{R})=$ $\operatorname{DP}_{V H}(\mathcal{R})=\emptyset$. We use the relations $\geq$ and $>$ generated by

$$
[a]=1 \quad[b]=0 \quad[f](x)=[g](x)=[G(x)]=x
$$

(over $\mathbb{N}$ ), and $\mathrm{P}_{U R}$ to remove the rule in $\mathrm{DP}_{H}(\mathcal{R})$ from $\tau_{H}=\left(\mathrm{DP}_{H}(\mathcal{R}), \emptyset, \mathcal{R}, e\right)$, thus proving operational termination of $\mathcal{R}$. Note that $>$ is monotonic, stable, transitive, and well-founded, and we have $\rightarrow_{\mathcal{R}} \subseteq>$ (and also $\rightarrow_{\mathcal{R}}^{+} \subseteq>$ ); the crucial point is that no substitution $\sigma$ satisfies $\sigma(f(x)) \rightarrow_{\mathcal{R}}^{*} \sigma(x)$ for the conditional rules: since $f(x)$ and $x$ do not unify, we should have $\sigma(f(x)) \rightarrow_{\mathcal{R}}^{+} \sigma(x)$ and hence $\sigma(f(x))>\sigma(x)$. But $[\sigma(f(x))]=\sigma(x) \ngtr \sigma(x)=[\sigma(x)]$. Thus, we do not need to ensure that $[\sigma(g(x))]>[\sigma(g(a))]$ holds! However, $[x]=x \geq x=[f(x)]$.

\section{Related Work and Conclusions}

To the best of our knowledge, this is the first correct and complete characterization of operational termination of deterministic 3-CTRS which is based on the notion of dependency pair. The notion of minimal operationally nonterminating term and the properties explored here (Sect.3) are also new in the literature. Furthermore, our treatment of the problem provides a bidimensional method that we have shown useful to simplify the analysis of operational termination 
itself and also to prove other termination properties like nontermination of 3CTRSs and termination of 2-CTRSs. The analysis of termination of 2-CTRSs can also be accomplished as termination of the underlying TRS (i.e., the TRS $\mathcal{R}_{u}$ which is obtained by just dropping the conditional part of the rules). However, in contrast to our Theorem 5, the analysis of termination of 2-CTRSs $\mathcal{R}$ as termination of the underlying TRS $\mathcal{R}_{u}$ provides a sufficient condition only; it may fail in those cases where taking into account the conditions of the rules is essential to prove termination. For instance the one rule 2-CTRS $a \rightarrow a \Leftarrow a \rightarrow b$ is terminating but $\mathcal{R}_{u}$ is not. We prove $\left(\operatorname{DP}_{H}(\mathcal{R}), \emptyset, \mathcal{R}, e\right)=(\{A \rightarrow A \Leftarrow a \rightarrow b\}, \emptyset, \mathcal{R}, e)$ finite (and hence $\mathcal{R}$ terminating) using $\mathrm{P}_{U R}$ with the removal triple $(\geq, \geq,>)$ generated by the interpretation $[a]=0$ and $[b]=1$ to remove $A \rightarrow A \Leftarrow a \rightarrow b$.

The recent Conditional Dependency Pairs (CDPs) by Nakamura et al. [13] apply to a subclass of 1 -CTRSs where the conditions $c$ in 1-rules $(\ell \rightarrow r \Leftarrow c)$ are terms instead of sequences $s_{1} \rightarrow t_{1}, \ldots, s_{n} \rightarrow t_{n}$. An instance $\sigma(c)$ of $c$ is satisfied if and only if $\sigma(c) \rightarrow^{*}$ true. We generate a (usually strict) subset of the pairs considered in [13, Definition 3.1]: $\mathrm{DP}_{H}(\mathcal{R}) \cup \operatorname{DP}_{V}(\mathcal{R}) \cup \operatorname{DP}_{V H}(\mathcal{R}) \subseteq C D P(\mathcal{R})$. Their chains [13, Definition 3.2] are also different to ours (Definition 5).

As remarked in the introduction, existing tools for proving termination of conditional TRSs currently use transformation techniques. We are not aware of any implementation of direct methods. The transformation which is typically used for this purpose is $\mathcal{U}$ in [14, Definition 7.2.48]. This transformation is not complete, however. For instance, $\mathcal{U}(\mathcal{R})$ is not terminating for $\mathcal{R}$ in Examples 1 and 20, but we proved them operationally terminating in Examples 19 and 20. Furthermore, when $\mathcal{U}(\mathcal{R})$ is terminating, tools may fail to find a proof. This is often due to the loss of information introduced by transformations, and also to the presence of new symbols and rules that prevent the search process from finding a proof. The techniques presented in this paper have been incorporated in the latest version of the tool MU-TERM ${ }^{1}$ [2]. The first benchmarks of existing examples in the literature are very positive and show that the 2D DP framework permits simple and fast proofs like the ones in the examples of this paper. This makes these techniques available to tools like MTT [4], which use MU-TERM as a backend for achieving proofs of operational termination of more general theories like membership equational programs or order-sorted rewrite theories. Direct termination methods for these wider logics will require extending the techniques presented here to the case of order-sorted conditional rewrite theories with types and subtypes, and where rewriting is context-sensitive and can take place modulo axioms $B$. This is envisaged as an interesting subject for future work.

\section{References}

1. Arts, T., Giesl, J.: Termination of term rewriting using dependency pairs. Theor. Comput. Sci. 236(1-2), 133-178 (2000)

2. Alarcón, B., Gutiérrez, R., Lucas, S., Navarro-Marset, R.: Proving termination properties with MU-TERM. In: Johnson, M., Pavlovic, D. (eds.) AMAST 2010. LNCS, vol. 6486, pp. 201-208. Springer, Heidelberg (2011)

\footnotetext{
${ }^{1}$ We thank Raúl Gutiérrez for the implementation of the 2D DP Framework.
} 
3. Clavel, M., Durán, F., Eker, S., Lincoln, P., Martí-Oliet, N., Meseguer, J., Talcott, C. (eds.): All About Maude - A High-Performance Logical Framework. LNCS, vol. 4350. Springer, Heidelberg (2007)

4. Durán, F., Lucas, S., Meseguer, J.: MTT: the Maude termination tool (system description). In: Armando, A., Baumgartner, P., Dowek, G. (eds.) IJCAR 2008. LNCS (LNAI), vol. 5195, pp. 313-319. Springer, Heidelberg (2008)

5. Futatsugi, K., Diaconescu, R.: CafeOBJ Report. AMAST Series. World Scientific, Singapore (1998)

6. Giesl, J., Arts, T.: Verification of Erlang processes by dependency pairs. Appl. Algebra Eng. Commun. Comput. 12, 39-72 (2001)

7. Giesl, J., Thiemann, R., Schneider-Kamp, P.: The dependency pair framework: combining techniques for automated termination proofs. In: Baader, F., Voronkov, A. (eds.) LPAR 2004. LNCS (LNAI), vol. 3452, pp. 301-331. Springer, Heidelberg (2005)

8. Giesl, J., Thiemann, R., Schneider-Kamp, P., Falke, S.: Mechanizing and improving dependency pairs. J. Autom. Reason. 37(3), 155-203 (2006)

9. Giesl, J., Thiemann, R., Schneider-Kamp, P.: Proving and disproving termination of higher-order functions. In: Gramlich, B. (ed.) FroCos 2005. LNCS (LNAI), vol. 3717, pp. 216-231. Springer, Heidelberg (2005)

10. Giesl, J., Schneider-Kamp, P., Thiemann, R.: AProVE 1.2: automatic termination proofs in the dependency pair framework. In: Furbach, U., Shankar, N. (eds.) IJCAR 2006. LNCS (LNAI), vol. 4130, pp. 281-286. Springer, Heidelberg (2006)

11. Kusakari, K., Nakamura, M., Toyama, Y.: Argument filtering transformation. In: Nadathur, G. (ed.) PPDP 1999. LNCS, vol. 1702, pp. 47-61. Springer, Heidelberg (1999)

12. Lucas, S., Marché, C., Meseguer, J.: Operational termination of conditional term rewriting systems. Inf. Process. Lett. 95, 446-453 (2005)

13. Nakamura, M., Ogata, K., Futatsugi, K.: On proving operational termination incrementally with modular conditional dependency pairs. Int. J. Comput. Sci. 40, 2 (2013)

14. Ohlebusch, E.: Advanced Topics in Term Rewriting. Springer, New York (2002)

15. Schernhammer, F., Gramlich, B.: Characterizing and proving operational termination of deterministic conditional term rewriting systems. J. Logic Algebr. Program. 79, 659-688 (2010)

16. Schernhammer, F., Gramlich, B.: VMTL-a modular termination laboratory. In: Treinen, R. (ed.) RTA 2009. LNCS, vol. 5595, pp. 285-294. Springer, Heidelberg (2009) 